\title{
Nonballistic heat conduction in an integrable random-exchange Ising chain studied with quantum master equations
}

\author{
Yonghong Yan, ${ }^{1}$ Chang-Qin Wu, ${ }^{1,2}$ Giulio Casati, $, 13,4$ Tomaž Prosen, ${ }^{5}$ and Baowen $\mathrm{Li}^{1,6, *}$ \\ ${ }^{1}$ Department of Physics and Centre for Computational Science and Engineering, National University of Singapore, \\ Singapore 117542, Republic of Singapore \\ ${ }^{2}$ Department of Physics, Fudan University, Shanghai 200433, People's Republic of China \\ ${ }^{3}$ Center for Nonlinear and Complex Systems, Università degli Studi dell'Insubria, via Valleggio 11, Como 22100, Italy \\ ${ }^{4}$ CNISM, CNR-INFM, and Istituto Nazionale di Fisica Nucleare, Sezione di Milano \\ ${ }^{5}$ Physics Department, Faculty of Mathematics and Physics, University of Ljubljana, 1000 Ljubljana, Slovenia \\ ${ }^{6}$ NUS Graduate School for Integrative Sciences and Engineering, Singapore 117597, Republic of Singapore \\ (Received 6 January 2008; revised manuscript received 9 April 2008; published 23 May 2008)
}

\begin{abstract}
We numerically investigate the heat conduction in a random-exchange Ising spin chain by using the quantum master equation. The chain is subject to a uniform transverse field $h$, while the exchange couplings $\left\{Q_{n}\right\}$ between the nearest neighbor spins are random; the largest size we simulate is up to 10 . This model is integrable; i.e., the nearest neighbor level spacing distribution is Poissonian. However, we find clear evidence of nonballistic transport. In the small coupling regime $\left(Q_{n} \ll h\right)$, an energy and/or temperature gradient in the bulk of the system is observed and the energy current appears to be proportional to the inverse of the system size. Moreover, we find that in the low and high temperature regimes, the thermal conductivity $\kappa$ and the specific heat $C_{v}$ have the same dependence on temperature. The large coupling case $\left(Q_{n} \sim h\right)$ is also discussed.
\end{abstract}

DOI: 10.1103/PhysRevB.77.172411

PACS number(s): 05.60.Gg, 05.30.-d, 05.70.Ln

\section{INTRODUCTION}

The derivation of Fourier's law of heat conduction from the microscopic dynamics without any ad hoc statistical assumption is one of the great challenges of nonequilibrium statistical mechanics. ${ }^{1}$ Even in the context of classical dynamical systems, the issue of energy (heat) transport, in spite of having a long history (recently reviewed in Ref. 2), is not completely settled. In the harmonic chain, no global thermal gradient occurs due to the lack of scattering between modes. ${ }^{3}$ On the other hand, some classical nonlinear systems of interacting particles, especially those with nonlinear on-site potentials, ${ }^{4}$ typically exhibit a diffusive behavior above a critical interaction strength, which then leads to the onset of Fourier's law, $J=-\kappa \nabla T$, relating the macroscopic heat flux to the temperature gradient.

However, much less is known about transport in quantum systems. The main problem is that, unlike with the classical systems, time and space computer requirements for numerical simulations exponentially increase with the system size. As a consequence, investigations have been so far mainly focused on the linear response theory, ${ }^{5,6}$ whose validity is questionable. ${ }^{7}$ We recall that in integrable systems such as one-dimensional spin-1/2 Heisenberg chains, due to the existence of nontrivial conservation laws, the current-current correlation functions typically do not decay to zero, which implies ballistic transport., 5 Therefore, quantum nonintegrability seems to be a necessary condition for normal conduction. $^{9-13}$

The interesting question then arises whether the randomness (or disorder) in an integrable system can induce normal (diffusive) transport. This problem is quite difficult even in the classical regime. For example, it has been shown that the energy transport in a mass-disordered harmonic chain strongly depends on the spectral properties of the thermal baths and, for some particular situation, even normal thermal conductivity has been found. ${ }^{14,15}$ The heat conduction in a quantum harmonic chain, with random couplings, has been recently considered ${ }^{16}$ and it has been found that a finite temperature gradient can be created. In this Brief Report, we study the heat conduction in a random integrable Ising chain by using the quantum master equation (QME). We find a clear evidence of nonballistic transport and also an indication of diffusive transport.

This Brief Report is organized as follows: In Sec. II, we describe the model and the method. Then in Sec. III, we present the numerical results. Section IV is devoted for the summary.

\section{MODEL AND METHOD}

We consider a spin-1/2 Ising chain subjected to a uniform transverse magnetic field $h \cdot{ }^{17-19}$ The Hamiltonian reads

$$
H=\sum_{n=1}^{N} h \sigma_{n}^{z}-\sum_{n=1}^{N-1} Q_{n} \sigma_{n}^{x} \sigma_{n+1}^{x},
$$

where $N$ is the number of spins, the operators $\sigma_{n}^{x}$ and $\sigma_{n}^{z}$ are the Pauli matrices for the $n$th spin, and $\left\{Q_{n}\right\}$ are the random numbers to be specified. In fact, system (1) is integrable as can be mapped into a model of free fermions through a Jordan-Wigner transformation. In particular, the nearest neighbor level spacing distribution $P(s)$ is Poissonian, $P_{P}(s)=\exp (-s)$, which is in contrast to the Wigner distribution $\left[P_{W}=(\pi s / 2) \exp \left(-\pi s^{2} / 4\right)\right]$ expected for chaotic systems. In Fig. 1, we show the nearest neighbor level spacing distribution $P(s)$ for two different cases; in both cases, the distributions are very close to the Poisson distribution as expected. Here, we take $h=1$ and choose a uniform distribution for the exchange couplings, i.e., $Q_{n} \in(0.05,0.15)$ in Fig. 1(a) 


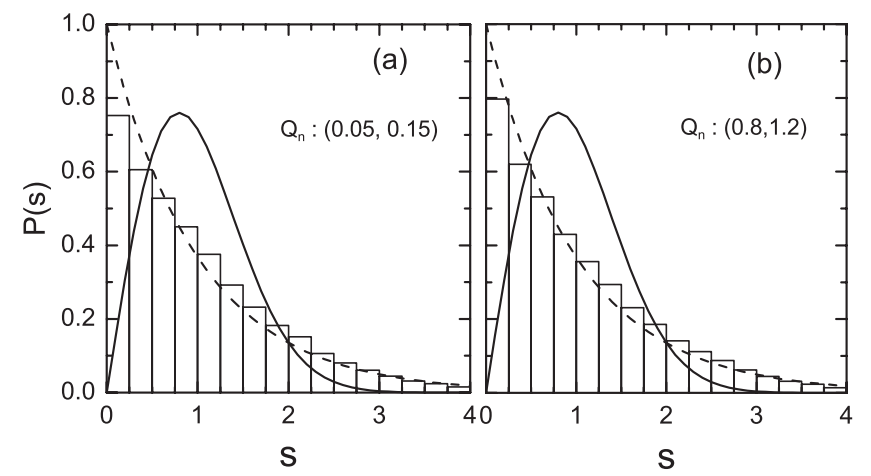

FIG. 1. Nearest neighbor level spacing distribution $P(s)$ for two different cases. In both (a) and (b), we take $h=1$. In (a), the couplings $\left\{Q_{n}\right\}$ are uniformly distributed in an interval, i.e., $Q_{n}$ $\in(0.05,0.15)$, while in (b), $Q_{n} \in(0.8,1.2)$. The size is $N=10$, and sample is taken over 30 realizations of $\left\{Q_{n}\right\}$. As a comparison, we also show the Poisson distribution (dashed line) and the Wigner distribution (full line).

and $Q_{n} \in(0.8,1.2)$ in Fig. 1(b). These parameters will be used in the following discussion. However, we should mention that one can, actually, choose other random distribution of $\left\{Q_{n}\right\}$, e.g., the Gaussian distribution. Here, we use a simple uniform distribution since we focus on whether randomness (disorder) can induce normal transport in an integrable model.

In the following, we use the QME to study heat transport in system (1). To this end, we need to couple both ends of the chain to thermal baths with different temperatures. However, for convenience, we illustrate the method with just one bath coupled to the left end of the chain. Then, the Hamiltonian (including one bath) can be written as

$$
\mathcal{H}=H+H_{R}+V
$$

where $H_{R}=\Sigma_{k} \hbar \omega_{k} a_{k}^{\dagger} a_{k}$ is a phonon bath, with $a_{k}^{\dagger}\left(a_{k}\right)$ as the phonon creation (annihilation) operator, and $V=\sigma_{1}^{x} \Sigma_{k}\left(g_{k} a_{k}\right.$ $\left.+g_{k}^{*} a_{k}^{\dagger}\right) \equiv \sigma_{1}^{x} R$ is the interaction between the chain and the bath. Under the assumption that the coupling between the chain and the bath is weak, we can derive an evolution equation for the system density matrix $\rho(\hbar=1)$ in the Markovian approximation, ${ }^{20}$

$$
\frac{d \rho}{d t}=-i[H, \rho]+\left(\left[W \rho, \sigma_{1}^{x}\right]+\text { H.c. }\right),
$$

where $W=\int_{0}^{\infty} g(\tau) \sigma_{1}^{x}(-\tau) d \tau, \quad \sigma_{1}^{x}(t)=e^{i H t} \sigma_{1}^{x} e^{-i H t}, \quad$ and $g(t)$ $=\operatorname{Tr}\left[e^{-\beta H_{R}} R(t) R\right] / \operatorname{Tr}\left(e^{-\beta H_{R}}\right)$ are the equilibrium correlation functions of the bath.

If the couplings $\left\{Q_{n}\right\}$ are much smaller than the field $h$, i.e., $Q_{n} \ll h$, then we can neglect the interaction term in $H$ when evaluating $W$. This finally leads to a Lindblad-type equation, ${ }^{21,22}$

$$
\frac{d \rho}{d t}=-i[H, \rho]+\mathcal{L} \rho,
$$

where

$$
\begin{aligned}
\mathcal{L} \rho= & \frac{\Gamma^{-}}{2}\left(2 \sigma_{1}^{-} \rho \sigma_{1}^{+}-\rho \sigma_{1}^{+} \sigma_{1}^{-}-\sigma_{1}^{+} \sigma_{1}^{-} \rho\right) \\
& +\frac{\Gamma^{+}}{2}\left(2 \sigma_{1}^{+} \rho \sigma_{1}^{-}-\rho \sigma_{1}^{-} \sigma_{1}^{+}-\sigma_{1}^{-} \sigma_{1}^{+} \rho\right)
\end{aligned}
$$

is a dissipative term with $\Gamma^{+}=\left.2 n(\omega) \mathcal{J}(\omega)\right|_{\omega=2 h} \equiv \alpha n(2 h)$ and $\Gamma^{-}=\left.2[1+n(\omega)] \mathcal{J}(\omega)\right|_{\omega=2 h} \equiv \alpha[1+n(2 h)]$. Here, $n(\omega)=\left(e^{\omega / T}\right.$ $-1)^{-1}$ is the Bose-Einstein distribution function $\left(k_{B}=1\right)$ and $\mathcal{J}(\omega)=\pi \Sigma_{k}\left|g_{k}\right|^{2} \delta\left(\omega-\omega_{k}\right)$ is the bath spectral function. Since only $\mathcal{J}$ s value at $2 h$ is relevant, we denote $\left.2 \mathcal{J}(\omega)\right|_{\omega=2 h}$ with $\alpha$. We note that in this weak coupling case, it does not matter whether the bath is Ohmic or not.

However, if the couplings are not sufficiently small, we need to evaluate $W$ in the representation of the eigenstates $\{|n\rangle\}$ of $H$ with $H|n\rangle=\varepsilon_{n}|n\rangle$. Then, the quantum master equation reads ${ }^{9,20}$

$$
\frac{d \rho}{d t}=-i[H, \rho]+\left(\left[X \rho, \sigma_{1}^{x}\right]+\text { H.c. }\right),
$$

where the operator $X$ is defined by $\langle m|X| n\rangle$ $=\lambda \varepsilon_{m, n} n\left(\varepsilon_{m, n}\right)\left\langle m\left|\sigma_{1}^{x}\right| n\right\rangle$, with $\varepsilon_{m, n} \equiv \varepsilon_{m}-\varepsilon_{n}$. Here, the bath spectral function we use is of the Ohmic type with a large cutoff $\omega_{c}$, i.e., $\mathcal{J}(\omega)=\lambda \omega\left(\omega<\omega_{c}\right)$. One can easily check that the canonical distribution $\rho=e^{-\beta H} / \operatorname{Tr}\left(e^{-\beta H}\right)$ is a solution of Eq. (6) with $\beta$ as the inverse of the bath temperature. The master equation [Eq. (4) or (6)] was solved by the fourthorder Runge-Kutta method. In numerical simulations, we use the Lindblad formalism for the weak coupling case and the general QME [Eq. (6)] for the strong coupling case. Also, we fix the magnetic field $h=1$ (as the unit of energy) and $\alpha=\lambda=0.01$. The simulation time was chosen to be long enough such that the final density matrix $\rho$ no longer appreciably changes with time, and thus, represents a steady state.

\section{NUMERICAL RESULTS}

First, we perform equilibrium simulations with the Lindblad equation [Eq. (4)] (see also Ref. 22). To this end, we set the left and the right baths to the same temperature $T$ and the coupling constants uniformly distributed in the interval $Q_{n}$ $\in(0.05,0.15)$. In Fig. 2 , we plot the numerical results for the local energy $E\left(\equiv E_{2}\right)$, which were averaged over 30 realizations of $\left\{Q_{n}\right\}$ versus the temperature for a chain with $N=4$. The local energy density is defined as $E_{n}=\operatorname{Tr} \rho H_{n}$, where $H_{n}$ is the local energy density operator,

$$
H_{n}=-Q_{n} \sigma_{n}^{x} \sigma_{n+1}^{x}+\frac{h}{2}\left(\sigma_{n}^{z}+\sigma_{n+1}^{z}\right) .
$$

At a low temperature, $E$ saturates to a constant that is determined by the ground state. For larger $T$, numerical results give $E \sim-1 / T$ (which means the specific heat $C_{v}=\frac{\partial E}{\partial T} \sim T^{-2}$ ). The central local energy can also be calculated by using the canonical average, $\left\langle\mathrm{H}_{2}\right\rangle=\operatorname{Tr}\left(\mathrm{H}_{2} e^{-\beta H_{2}}\right) / \operatorname{Tr} e^{-\beta H_{2}}$, which is shown in Fig. 2 (solid line). We observe that the two curves nearly perfectly fit when the couplings $\left\{Q_{n}\right\}$ are small. However, when $\left\{Q_{n}\right\}$ become larger, e.g., $Q_{n} \in(0.8,1.2)$, the difference emerges (see inset of Fig. 2). This is because the 


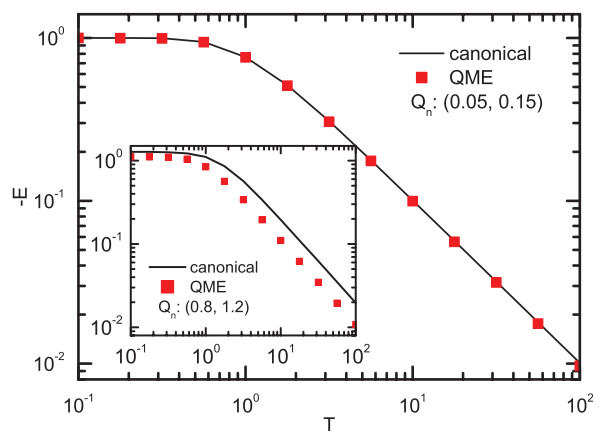

FIG. 2. (Color online) Local energy $E\left(\equiv E_{2}\right)$ as a function of temperature $T$ for a random system with $N=4$, and the couplings $Q_{n} \in(0.05,0.15)$. Each point (square) corresponds to equilibrium simulations in which the bath temperatures are set to the same value $T$. The solid line corresponds to the canonical average $\left\langle H_{2}\right\rangle$ $=\operatorname{Tr}\left(H_{2} e^{-\beta H_{2}}\right) / \operatorname{Tr} e^{-\beta H_{2}}$. The sampling is taken over 30 realizations. In the inset, the same data are shown for the couplings $Q_{n}$ $\in(0.8,1.2)$.

Lindblad equation [Eq. (4)] is only valid for small $\left\{Q_{n}\right\}$.

In order to study the heat conduction properties, we also need to define the local temperature. In a classical lattice model, one can define the local temperature via the average kinetic energy. In our quantum model, the situation is not so obvious even in the equilibrium case. ${ }^{23}$ In this Brief Report, we use the local bond energy $E_{n}=\operatorname{Tr} \rho H_{n}$ to identify the local temperature. If the couplings are small, one can expect a factorized form of the density matrix, $\rho \sim \otimes_{n} e^{-\beta_{n} H_{n}}$, with $\beta_{n}$ as the local temperature. Then, there is one-to-one correspondence between the local temperature and the bond energy.

Now, we turn to discuss heat conduction in the Ising chain with random couplings. We set the couplings uniformly distributed in the interval $Q_{n} \in(0.05,0.15)$. Figure 3 shows the energy profile for nonequilibrium simulations of a random chain, with different sizes, ranging from $N=6$ to $N=10$. The temperatures of the baths are set to $T_{L}=10$ and $T_{R}=5$. We observe that in the bulk of the chain, an energy gradient is built for any system size. For comparison, we also show the energy profile for an ordered chain wherein the energy gradient vanishes, thus, signaling ballistic transport.

The energy current operator can be defined through the equation of continuity $\partial_{t} H_{n}=i\left[H, H_{n}\right]=J_{n}-J_{n+1}$. In our case,

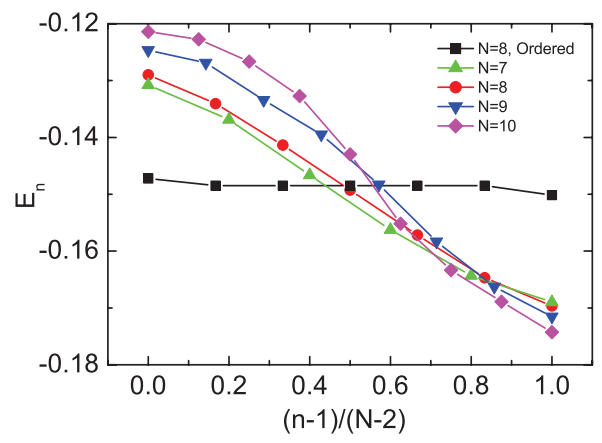

FIG. 3. (Color online) Energy profile $E_{n}$ for a random chain with $Q_{n} \in(0.05,0.15)$ and different sizes $N$. The numerical data are averaged over 50 realizations of $\left\{Q_{n}\right\}$. The temperatures of the left and right baths are $T_{L}=10$ and $T_{R}=5$. For comparison, the energy profile $E_{n}$ of an ordered chain with $Q_{n}=Q=0.1$ is also shown (squares).

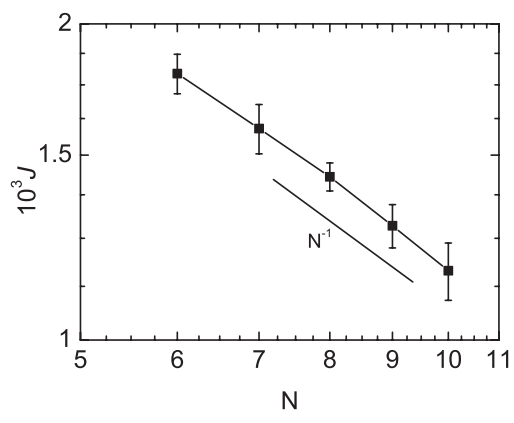

FIG. 4. Double-logarithmic plot of the heat current as a function of size. $Q_{n} \in(0.05,0.15)$. $T_{L}=10$ and $T_{R}=5$. The sampling is taken over 30 realizations; the error bar represents the standard error of the mean value.

$$
J_{n}=h\left[Q_{n} \sigma_{n+1}^{x}-Q_{n-1} \sigma_{n-1}^{x}\right] \sigma_{n}^{y} .
$$

In Fig. 4, we plot the energy current $J=\operatorname{Tr} \rho J_{n}$ (which does not depend on $n$ in the steady state). The results suggest that the current is proportional to the inverse of the system size, $J \sim N^{-1}$

In Fig. 5, we plot the thermal conductivity $\kappa=J /\left(T_{L}\right.$ $\left.-T_{R}\right)$ as a function of temperature. In the numerical simulations, we set the temperatures of the baths as $T_{L}=T(1+\epsilon)$ and $T_{R}=T(1-\epsilon)$ with $\epsilon=0.02$. It turns out that the thermal conductivity $\kappa$ and the specific heat $c_{v}$ have a similar dependence on temperature. Indeed, since in our case the couplings $\left\{Q_{n}\right\}$ are very small, the specific heat of a single spin can be approximated by $c_{v} \simeq\left(\frac{2 h}{T}\right)^{2} e^{-2 h T /}\left(1+e^{-2 h T}\right)^{2}$. That means that in the low temperature regime, $c_{v} \sim\left(\frac{2 h}{T}\right)^{2} e^{-2 h / T}$, while in the high temperature regime, $c_{v} \sim T^{-2}$. The inset of Fig. 5 shows that, in fact, the low temperature behavior of thermal conductivity is $\kappa \sim\left(\frac{2 h}{T}\right)^{2} e^{-2 h / T}$. Instead, at a high temperature, our numerical results show that $\kappa$ roughly decays as $\kappa$ $\sim T^{-2}$, which is similar to the behavior of a classical Heisenberg model. ${ }^{24}$

Finally, we have investigated the thermal conductivity for a large coupling case $Q_{n} \in(0.8,1.2)$. Here, the numerical difficulties are even more severe. Our results, as shown in Fig. 6 , indicate that an energy gradient is built if the temperature difference of the baths is large enough (inset). Moreover, at a

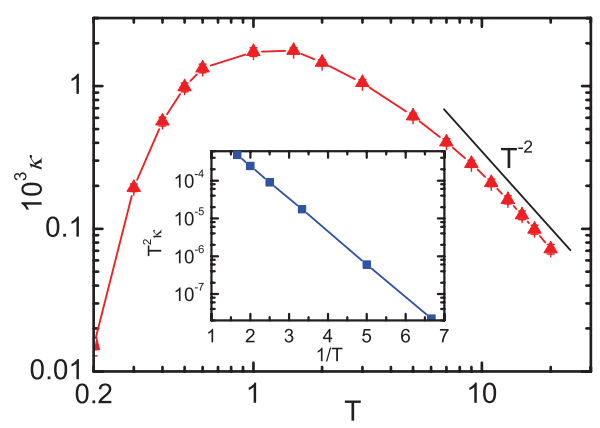

FIG. 5. (Color online) Thermal conductivity $\kappa$ as a function of temperature $T$ for $N=8$ and $Q_{n} \in(0.05,0.15)$. The bath temperatures are $T_{L}=T(1+\epsilon)$ and $T_{R}=T(1-\epsilon)(\epsilon=0.02)$. The error bars represent the standard error of the mean value. The inset shows the low temperature behavior of thermal conductivity. 


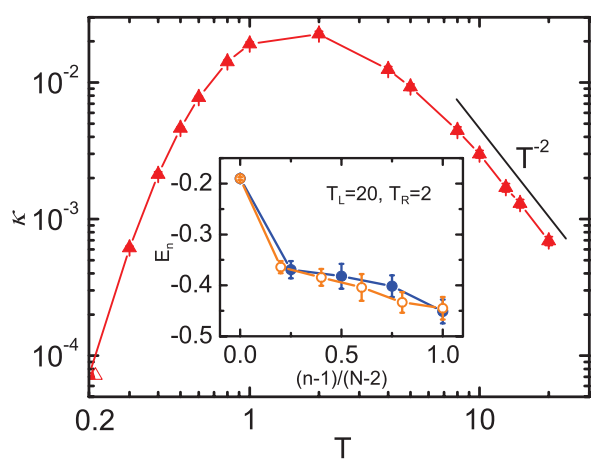

FIG. 6. (Color online) Thermal conductivity $\kappa$ as a function of temperature $T$ (triangles). We set the temperatures of the baths as $T_{L}=T(1+\epsilon)$ and $T_{R}=T(1-\epsilon)(\epsilon=0.02) . Q_{n} \in(0.8,1.2), N=6$. The inset shows the energy profile with $T_{L}=20, T_{R}=2, N=6$ (solid circles), and $N=7$ (open circles). The error bar shows the standard error.

high temperature, $\kappa$ decays as $\kappa \sim T^{-2}$, while no definite conclusions can be made for the low temperature behavior of thermal conductivity.

\section{SUMMARY}

In summary, we have studied heat conduction in a random Ising spin chain within the quantum master equation formal- ism. The chain is subject to a uniform magnetic field $h$ and the couplings $\left\{Q_{n}\right\}$ between the nearest neighboring spins are random. This is an integrable model and, in particular, the nearest neighbor level spacing distribution is of Poisson type. However, we find evidence of nonballistic, perhaps, diffusive transport. Indeed, in the small coupling case $\left(Q_{n} \ll h\right)$, a nonvanishing energy and/or temperature gradient in the bulk of the system is observed and the energy current is approximately proportional to the inverse of the system size.

Note, however, that due to the small system sizes that we were only able to simulate, the energy gradient in the center of the system seems to be increasing with the system size (Fig. 3). Therefore, we also cannot exclude the possibility of an insulating behavior, namely, that the bulk conductivity decreases with the system size. This would be consistent with the possibility of many-body localization observed or suggested in related contexts. ${ }^{25,26}$

\section{ACKNOWLEDGMENTS}

This work was supported by an Academic Research Fund under Grant No. R-144-000-203-112 from Ministry of Education of Republic of Singapore, MOE of China (Grant No. B06011), NSFC, and ARRS (Slovenia) under Grants No. P1-0044 and No. J1-7347.
*Corresponding author; phylibw@nus.edu.sg

${ }^{1}$ F. Bonetto, J. L. Lebowitz, and L. Rey-Bellet, in Mathematical Physics 2000, edited by A. Fokas, A. Grigoryan, T. Kibble, and B. Zegarlinsky (Imperial College, London, 2000), p. 128.

${ }^{2}$ S. Lepri, R. Livi, and A. Politi, Phys. Rep. 377, 1 (2003).

${ }^{3}$ Z. Rieder, J. L. Lebowitz, and E. Lieb, J. Math. Phys. 8, 1073 (1967); U. Zürcher and P. Talkner, Phys. Rev. A 42, 3278 (1990).

${ }^{4}$ B. Hu, B. Li, and H. Zhao, Phys. Rev. E 57, 2992 (1998); B. Hu, B. Li, and H. Zhao, ibid. 61, 3828 (2000); K. Aoki and D. Kusnezov, Phys. Lett. A 265, 250 (2000).

${ }^{5}$ X. Zotos, F. Naef, and P. Prelovšek, Phys. Rev. B 55, 11029 (1997)

${ }^{6} \mathrm{X}$. Zotos and P. Prelovšek, Interacting Electrons in Low Dimensions, Physics and Chemistry of Materials with LowDimensional Structures (Kluwer, Dordrecht, 2003); Strong Interactions in Low Dimensions, edited by D. Baeriswyl and L. Degiorgi (Kluwer, Dordrecht, 2004).

${ }^{7}$ J. Gemmer, R. Steinigeweg, and M. Michel, Phys. Rev. B 73, 104302 (2006), and references therein.

${ }^{8}$ A. V. Sologubenko, E. Felder, K. Giannò, H. R. Ott, A. Vietkine, and A. Revcolevschi, Phys. Rev. B 62, R6108 (2000); A. V. Sologubenko, K. Giannò, H. R. Ott, A. Vietkine, and A. Revcolevschi, ibid. 64, 054412 (2001).

${ }^{9}$ K. Saito, Europhys. Lett. 61, 34 (2003).

${ }^{10}$ C. Mejía-Monasterio, T. Prosen, and G. Casati, Europhys. Lett. 72, 520 (2005).

${ }^{11}$ M. Michel, M. Hartmann, J. Gemmer, and G. Mahler, Eur. Phys.
J. B 34, 325 (2003).

${ }^{12}$ M. Michel, G. Mahler, and J. Gemmer, Phys. Rev. Lett. 95, 180602 (2005).

${ }^{13}$ R. Steinigeweg, J. Gemmer, and M. Michel, Europhys. Lett. 75, 406 (2006).

${ }^{14}$ A. Dhar, Phys. Rev. Lett. 86, 5882 (2001).

${ }^{15}$ B. Li, H. Zhao, and B. Hu, Phys. Rev. Lett. 86, 63 (2001).

${ }^{16}$ C. Gaul and H. Büttner, Phys. Rev. E 76, 011111 (2007).

${ }^{17}$ E. Westerberg, A. Furusaki, M. Sigrist, and P. A. Lee, Phys. Rev. Lett. 75, 4302 (1995).

${ }^{18}$ G. Schehr and H. Rieger, Phys. Rev. Lett. 96, 227201 (2006).

${ }^{19}$ L. F. Santos, J. Phys. A 37, 4723 (2004).

${ }^{20}$ R. Kubo, M. Toda, and N. Hashitsume, Statistical Physics II (Springer-Verlag, New York, 1991).

${ }^{21}$ G. Lindblad, Commun. Math. Phys. 48, 119 (1976).

${ }^{22} \mathrm{H}$. Wichterich, M. J. Henrich, H. P. Breuer, J. Gemmer, and M. Michel, Phys. Rev. E 76, 031115 (2007).

${ }^{23}$ M. Hartmann, G. Mahler, and O. Hess, Phys. Rev. Lett. 93, 080402 (2004); J. Gemmer, M. Michel, and G. Mahler, Quantum Thermodynamics (Springer, New York, 2004), Vol. LNP657.

${ }^{24}$ A. V. Savin, G. P. Tsironis, and X. Zotos, Phys. Rev. B 72, 140402(R) (2005); A. V. Savin, G. P. Tsironis, and X. Zotos, ibid. 75, 214305 (2007)

${ }^{25}$ A. Karahalios, A. Metavitsiadis, X. Zotos, A. Gorczyca, and P. Prelovšek, arXiv:0803.1379v1 (unpublished).

${ }^{26}$ M. Žnidarič, T. Prosen, and P. Prelovšek, Phys. Rev. B 77, 064426 (2008); V. Oganesyan and D. A. Huse, ibid. 75, 155111 (2007). 